\title{
Foot Syndactyly: A Clinical and Demographic Analysis
}

\author{
Jong Ho Kim, Byung Jun Kim, Sung Tack Kwon \\ Department of Plastic and Reconstructive Surgery, Seoul National University College of Medicine, Seoul, Korea
}

Background Syndactyly of the foot is the second most common congenital foot anomaly. In East Asia, however, no large case study has been reported regarding the clinical features of isolated foot syndactyly. In this study, we report a review of 118 patients during the last 25 years.

Methods We conducted a chart review of patients who underwent surgical correction for foot syndactyly between January 1990 and December 2014. Operations were performed with a dorsal triangular flap and a full-thickness skin graft. The demographics of included patients and their clinical features were evaluated. Surgical outcomes and complications were analyzed. Results Among 118 patients with 194 webs (155 feet), 111 patients showed nonsyndromic cases and 7 patients showed syndromic cases. In 80 unilateral cases (72.1\%), the second web was the most frequently involved (37.5\%), followed by the fourth $(30 \%)$, the first $(15 \%)$, the third (15\%), the first and second in combination (1.3\%), and the second and third in combination (1.3\%). Among 31 bilateral cases, 2 cases were asymmetric. Among the remaining 29 symmetric bilateral cases, the second web was the most frequently involved (45.2\%), followed by the first $(22.6 \%)$, and the fourth (6.5\%). No specific postoperative complications were observed, except in the case of 1 patient $(0.51 \%)$ who required a secondary operation to correct web creep.

Conclusions This retrospective clinical study of 118 patients with both unilateral and bilateral foot syndactyly revealed that the second web was the most frequently involved. In addition, complete division and tension-free wound closure with a full-thickness skin graft of sufficient size showed good postoperative results.

Keywords Syndactyly / Foot deformities, congenital / Postoperative complications
Correspondence: Sung Tack Kwon Department of Plastic and Reconstructive Surgery, Seoul National University College of Medicine, 103 Daehak-ro, Jongno-gu, Seoul 03080, Korea

Tel: +82-2-2072-3759

Fax: +82-2-747-5130

E-mail: stk59@snu.ac.kr
The authors thank Kyu-Young Kim for the medical illustrations.

No potential conflict of interest relevant to this article was reported.

\section{INTRODUCTION}

Syndactyly of the hand or foot is a common congenital limb malformation with an incidence of approximately 1 in every 2,000 to 2,500 cases. It is characterized by a variable degree of fusion of the skin and/or bone of adjacent digits. As reported by
Castilla et al. [1] in 1980, isolated syndactyly has been observed in 2.2/10,000 livebirths, with isolated foot syndactyly occurring 4 times more frequently than isolated hand syndactyly. However, foot syndactyly has been less frequently discussed in the literature, despite its higher incidence compared with hand syndactyly. 
Castilla et al. [1] reported 103 cases of foot syndactyly from patients with isolated hand and foot syndactyly and Mondolfi [2] reported 43 patients with foot syndactyly. They first described the web distribution of isolated foot syndactyly. To the best of our knowledge, no further large studies presenting demographic analyses of foot syndactyly have been conducted since these 2 studies.

In this study, we report a review of 118 patients ( 155 feet) during a 25-year period. This is the first large study of foot syndactyly, analyzing more than 100 patients in East Asia. We present a demographic analysis, the clinical features, and postoperative outcomes of foot syndactyly.

\section{METHODS}

A chart review was conducted on patients who underwent surgical correction for foot syndactyly between January 1990 and December 2014, in the Department of Plastic and Reconstructive Surgery. The study was approved by the Institutional Review Board of our institution. Cases of polysyndactyly that did not require division surgery, syndactyly associated with constriction band syndrome, and trauma-induced acquired syndactyly were excluded.

Patients' demographic information, including age and sex, and the clinical features of syndactyly were evaluated, including features such as partial or complete, simple or complex, and unilateral or bilateral involvement. In nonsyndromic cases, the involved web distribution was analyzed. Functional outcome and postoperative complications such as web creep, flexion contractures, and angulation deformity were assessed. In referred cases, the causes of secondary surgery were analyzed based on medical records.

\section{Surgical technique}

All operations were performed by a single surgeon. The interdigital triangular flap was designed to reconstruct the web space in a modification of the methods originally described by Cronin [3] in 1956. The dorsal triangular skin flaps were marked from the point of the metatarsal head dimple to the interdigital crease. The triangle was designed such that the ratio of the height to the base was greater than 1.5 , so that it could reach the plantar interdigital crease. A zig-zag incision was designed to place the tip of the triangle at the joint level. The division site was overcorrected to prevent a secondary flexion contracture at the volar side. In this procedure, meticulous dissection was performed to avoid injury to the neurovascular bundle. Any tethering points found during physical examination were fully released.

The remaining skin defect was then covered using full-thick- ness skin (FTS) harvested from the inguinal area. The surgeon made an effort to obtain tension-free wound closure with a skin graft of sufficient size by estimating the amount of FTS in a fully released state. In cases of multiple web space involvement that required 2 or more operations, the next operation for the neighboring web space was delayed, with a mean interval of 9.5 months (range, 5-16 months).

\section{RESULTS}

Operations for syndactyly were performed on 194 webs (155 feet, 118 patients). Seven patients (13 feet, 36 webs) had cases of syndromic syndactyly; these consisted of 5 cases of Apert syndrome, 1 case of Klippel-Trenaunay-Weber syndrome, and 1 case of Van der Woude syndrome.

The demographic information and clinical features of 111 patients with nonsyndromic syndactyly ( 142 feet, 158 webs) were analyzed. The study population consisted of 59 male and 52 female patients (Table 1 ). Eighty patients $(72.1 \%)$ had unilateral syndactyly (right, 42; left, 38), and 31 patients (27.9\%) had bilateral syndactyly. Ten webs (6.3\%) arose from complex syndactyly with bone fusion, while 148 webs (93.7\%) were of the simple type. Eighty-five webs showed complete syndactyly (53.8\%) and 73 webs showed incomplete syndactyly (46.2\%). The age of the patients at the time of operation varied from 6 months to 17 years, with a median age of 15.1 months. The follow-up period ranged from 6 months to 12 years, with a median period of 3.1 years. Hand syndactyly was concurrent in 7 of 111 patients (6.3\%). In addition, all cases of foot syndactyly in the fourth web were concomitant with duplication of the fifth toe (Fig. 1).

In 80 cases of unilateral foot syndactyly, the first interdigital web was involved in 12 patients (15\%), the second in 30 patients (37.5\%), the third in 12 patients (15\%), the fourth in 24 patients (30\%), the first and second in 1 patient (1.3\%), and the second and third in 1 further patient (1.3\%).

Of the 31 patients with bilateral foot syndactyly, 29 patients showed symmetric and 2 patients showed asymmetric cases.

Table 1. The demographic information and clinical features of nonsyndromic patients

\begin{tabular}{|lc|}
\hline Particulars & No. \\
\hline Patients/Feet/Webs & $111 / 142 / 158$ \\
Sex (male:female) & $59: 52$ \\
Age at the first surgery & $15.1 \mathrm{mo}(6 \mathrm{mo}-17 \mathrm{yr})$ \\
Complete/Incomplete & $85 / 73$ \\
Bilateral/Unilateral & $31 / 80$ (right, 42; left, 38) \\
Simple/Complex & $148 / 10$ \\
Follow-up & $3.1 \mathrm{yr}(6 \mathrm{mo}-12 \mathrm{yr})$ \\
\hline
\end{tabular}




\section{Fig. 1. A case of foot syndactyly in the fourth web}

(A) Preoperative photograph. (B) X-ray image of the same patient. Even if duplication of the fifth toe was subtle on a physical examination, duplication was recognizable on X-ray (red arrow). (C) Postoperative photograph.

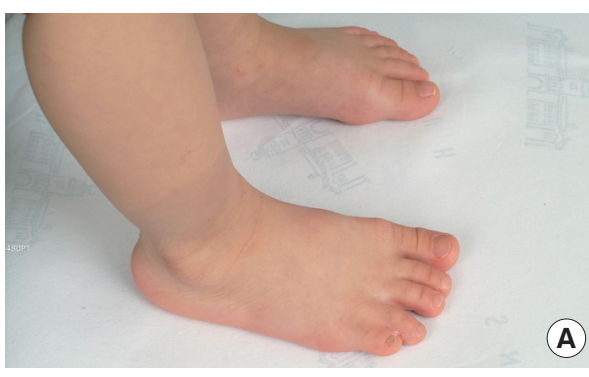

A)

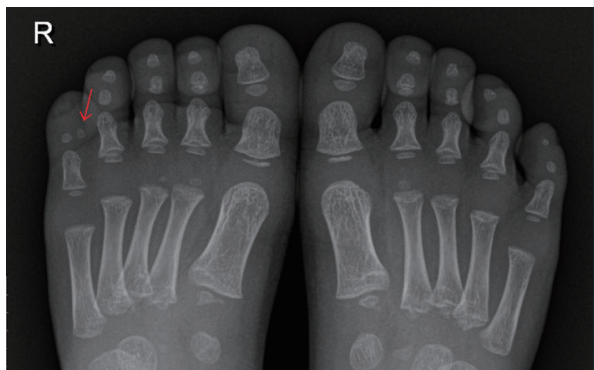

C

Fig. 2. Web distribution of patients

(A) Unilateral foot syndactyly (80 cases). (B) Bilateral symmetric foot syndactyly (29 cases).

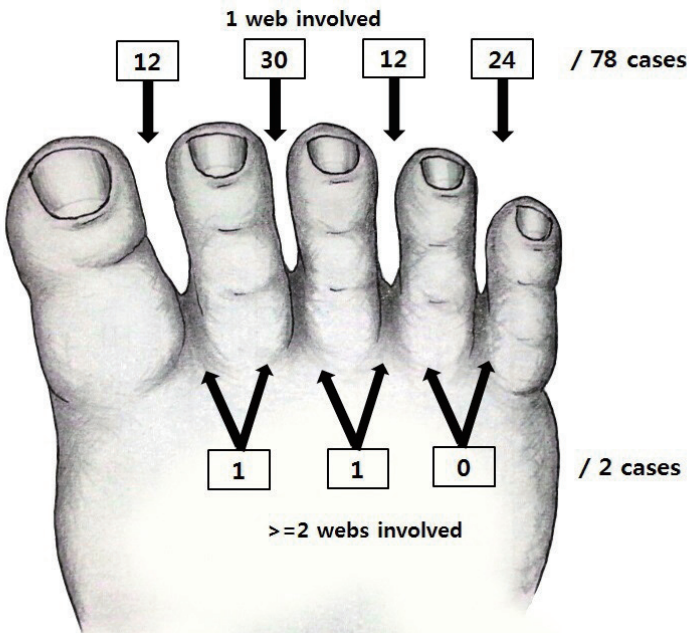

(A)

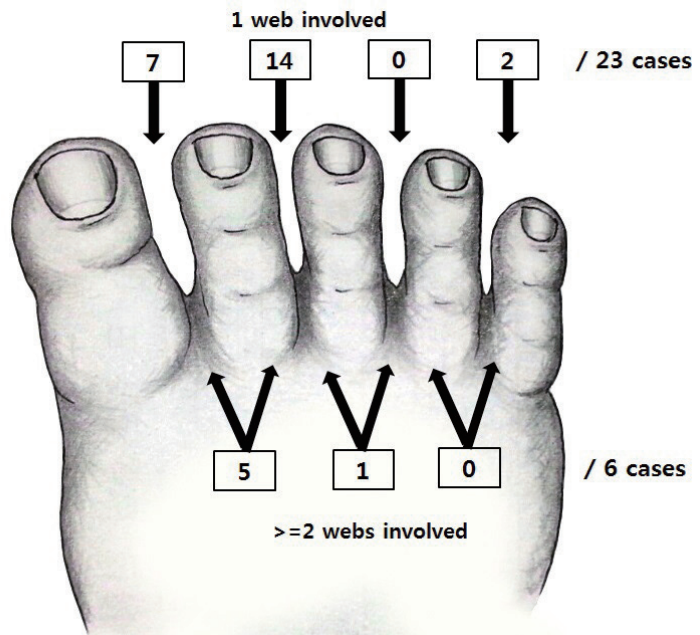

Among 29 cases of the symmetric bilateral type, the first interdigital web was involved in 7 patients $(22.6 \%)$, the second in 14 patients (45.2\%), the fourth in 2 patients $(6.5 \%)$, the first and second in 5 patients $(16.1 \%)$, and the second and third in 1 patient (3.2\%) (Fig. 2). In the 2 cases of bilateral asymmetric syndactyly, 1 patient had the first and second web space syndactyly on the right foot and the second web space on the left foot. The other bilateral asymmetric case involved the first web space on the right foot and the first and third web space on the left foot.

Of the 118 patients with syndactyly ( 155 feet, 194 webs) who received surgery, 3 patients (1.6\%) showed postoperative complications. One patient required secondary surgery to correct web creep (0.52\%). One patient had a hypertrophic scar $(0.52 \%)$, and another patient had a pressure sore of the heel $(0.52 \%)$ that was resolved with conservative management.

In addition, 6 of the 118 patients were referred from other hospitals due to secondary complications from syndactyly correction; there were 2 cases of total adhesion of the division site, 1 case of partial adhesion, 1 case of partial adhesion with angulation deformity, 1 case of hypertrophic scar, and 1 case of flexion contracture combined with hyperpigmentation (Table 2). All of these secondary cases were successfully corrected by the aforementioned surgical methods.

\section{DISCUSSION}

The incidence of hand or foot syndactyly is known to be 1 in every 2,000 to 2,500, which makes hand or foot syndactyly the 
second most common congenital limb malformation after polydactyly. Hand syndactyly frequently occurs in the third interdigital web space, while foot syndactyly most commonly occurs in the second interdigital web space. Isolated foot syndactyly is known to be more frequent than isolated hand syndactyly [1]. In spite of this higher incidence, there is less discussion of foot syndactyly in the literature. The minimal functional impairment that foot syndactyly imposes, as well as the lesser degree of aesthetic concern, may explain this. In East Asian cultures, however, it is common to go barefoot indoors. Therefore, those with foot syndactyly in East Asian cultures may experience comparatively greater psychological distress from the condition, resulting in a greater demand for surgical correction due to aesthetic concerns.

Castilla et al. [1] reported 103 cases of foot syndactyly out of 133 cases of hand or foot syndactyly. In this study, they reported that isolated syndactyly of the second and third toes was the most common type (52.6\%, 70/133 cases). Mondolfi [2] reported 43 patients with foot syndactyly, and of those, 23 pa-

\section{Table 2. Patients referred from other hospitals due to} postoperative complications

\begin{tabular}{|llll|}
\hline $\begin{array}{l}\text { Patient } \\
\text { no. }\end{array}$ & $\begin{array}{c}\text { Webspace } \\
\text { involved }\end{array}$ & \multicolumn{1}{c|}{ Complications } & $\begin{array}{c}\text { Previous } \\
\text { operation } \\
\text { technique }\end{array}$ \\
\hline 1 & Right third & $\begin{array}{l}\text { Partial adhesion+angulation } \\
\text { deformity }\end{array}$ & STSG from sole \\
2 & Left fourth & Partial adhesion & FTSG from groin \\
3 & Right third & Total adhesion & FTSG from groin \\
4 & Left second & Total adhesion & Open treatment \\
6 & Right second & Hypertrophic scar & FTSG from groin \\
\hline RTSG, split-thickness skin graft; FTSG, full-thickness skin graft. & FTSG from penis \\
\hline
\end{tabular}

tients had a fusion of the fourth and fifth toes with a duplication of the fifth toe. These 2 studies are the most recent studies that have included a demographic analysis of foot syndactyly cases. To the best of our knowledge, the 118 patients of this study constitute the largest sample of foot syndactyly cases among articles published since 1950 .

The term zygodactyly was first used by Weidenreich [4] in 1924 to describe simple, cutaneous webbing of the second and third toe. Zygodactyly is known to be the most common type of foot syndactyly [2,5]. Malik [6] proposed an extended set of 9 types of syndactyly based on genetics. Malik categorized zygodactyly as type 1-a, and a cutaneous web of the fourth web space as type 1-d, which are the first and second most common types of foot syndactyly, respectively. Data from our study is consistent with the results of these previous studies.

The surgical methods used for foot syndactyly were derived from those for hand syndactyly, which consist of division and reconstruction with a dorsal skin flap and a skin graft to cover the raw surface. Recently, various techniques that do not require the application of skin grafts, such as open treatment, primary closure by defatting $[7,8]$, or intricate local flaps $[9,10]$, have been reported. These techniques are advantageous in that they shorten the operation time and minimize donor site morbidity. However, these techniques, despite their advantages, are associated with increased risks of sensory deficit due to excessive subcutaneous fat removal for primary closure, wound dehiscence following extensive tension, and secondary complications, such as web creep, flexion contracture, and angulation deformity.

In our study, there were 6 secondary cases that were referred from other hospitals. These cases required divisional surgery with a split thickness skin graft (patient no. 1), open treatment (patient no. 4), and penile skin (patient no. 6) (Fig. 3). The use of split thickness skin on the defect site can lead to an angulation

\section{Fig. 3. Secondary cases}

(A) Preoperative photographs of patient no. 1. The patient underwent divisional surgery, and a split-thickness skin graft from the right sole was used for skin defect coverage. Partial adhesion of the division site and angulation deformity of the right third toe are shown. (B) Preoperative photograph of patient no. 4. The patient underwent syndactyly division by open treatment without a skin graft. Total adhesion had occurred despite the maintenance of interdigital dressing for more than 2 months postoperatively. (C) Preoperative photographs of patient no. 6 . The patient underwent a syndactyly operation with penile skin for coverage of the raw surface. Flexion contracture of the second toe and hyperpigmentation of the grafted area are shown.
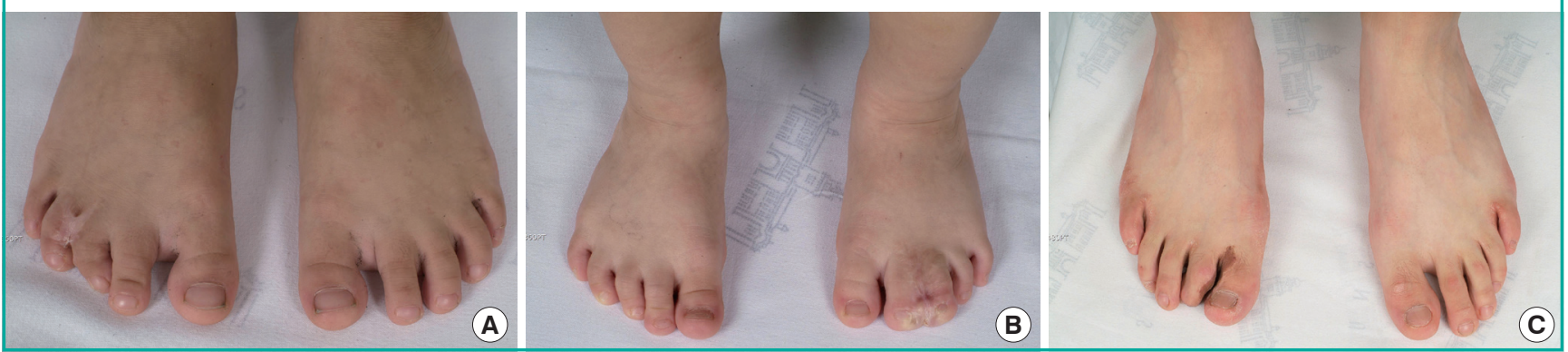
deformity or flexion contracture [11] and the use of penile skin can cause hyperpigmentation and flexion contracture [12]. Furthermore, open treatment poses a greater risk of adhesion of the division site. Therefore, complete division with a sufficient amount of FTS is important to reduce secondary deformity and overall medical cost.

We experienced 1 case requiring a secondary operation $(0.51 \%)$ due to web creep. The revision rate of our study was very low compared with that of previous studies, which reported revision rates up to $42 \%[11,13]$.

In conclusion, we were able to identify the demographic characteristics and clinical features of foot syndactyly in South Korea and the results are consistent with data from previous studies in Western countries. Foot syndactyly surgery using a dorsal skin flap and a sufficient FTS graft may be necessary for better postoperative outcomes and lower complication rates.

\section{REFERENCES}

1. Castilla EE, Paz JE, Orioli-Parreiras IM. Syndactyly: frequency of specific types. Am J Med Genet 1980;5:357-64.

2. Mondolfi PE. Syndactyly of the toes. Plast Reconstr Surg 1983;71:212-7.

3. Cronin TD. Syndactylism: results of zig-zag incision to prevent postoperative contracture. Plast Reconstr Surg (1946) 1956;18:460-8.

4. Weidenreich F. Die zygodactylie und ihre vererbung. Z Indukt Abstamm Vererbungsl 1924;32:304-11.
5. Temtamy SA, McKusick VA. Syndactyly. In: Bergsma D, editor. The genetics of hand malformations. New York: Alan R Liss; 1978. p.301-22.

6. Malik S. Syndactyly: phenotypes, genetics and current classification. Eur J Hum Genet 2012;20:817-24.

7. Hikosaka M, Ogata H, Nakajima T, et al. Advantages of open treatment for syndactyly of the foot: defining its indications. Scand J Plast Reconstr Surg Hand Surg 2009;43: 148-52.

8. Kawabata H, Ariga K, Shibata T, et al. Open treatment of syndactyly of the foot. Scand J Plast Reconstr Surg Hand Surg 2003;37:150-4.

9. Lim YJ, Teoh LC, Lee EH. Reconstruction of syndactyly and polysyndactyly of the toes with a dorsal pentagonal island flap: a technique that allows primary skin closure without the use of skin grafting. J Foot Ankle Surg 2007;46:86-92.

10. Itoh Y, Arai K. A new operation for syndactyly and polysyndactyly of the foot without skin grafts. Br J Plast Surg 1995; 48:306-11.

11. Percival NJ, Sykes PJ. Syndactyly: a review of the factors which influence surgical treatment. J Hand Surg Br 1989; 14: 196-200.

12. Mak AS, Poon AM, Tung MK. Use of preputial skin for the release of burn contractures in children. Burns 1995;21:3012.

13. Dao KD, Shin AY, Billings A, et al. Surgical treatment of congenital syndactyly of the hand. J Am Acad Orthop Surg 2004;12:39-48. 